\title{
The Italian Panoramic Monochromator
}

\section{F. Cavallini}

Osservatorio Astrofisico di Arcetri, Largo E. Fermi 5, I-50125 Firenze, Italy

Received June 17; accepted July 29, 1997

\begin{abstract}
A new instrument for solar bidimensional spectroscopy, the Italian Panoramic Monochromator (IPM), has been recently installed on the Télescope Heliographique pour l'Étude du Magnétisme et des Instabilités de l'atmosphère Solaire (THEMIS), the French-Italian solar telescope built in Tenerife (Canary Islands). On a square field $33^{\prime \prime} \times 33^{\prime \prime}$, this instrument allows one to obtain monochromatic images of the solar surface with high spectral resolution $(\mathcal{R} \geq 256000$ at $5500 \AA$ ), preserving all the spatial resolution delivered by the telescope $\left(\simeq 0.2^{\prime \prime}\right)$. The wavelength stability of the instrumental profile is very high, the maximum drift in 10 hours amounting to about $10 \mathrm{~ms}^{-1}$.
\end{abstract}

Key words: instrumentation: interferometers instrumentation: spectrographs - Sun: photosphere Sun: chromosphere

\section{Introduction}

The three-dimensional relationships between quantities like pressure, temperature, velocity and magnetic field, describing the state of the gas in different solar atmospheric structures, can only be investigated by narrow-band bidimensional spectroscopy.

For this reason, a new instrument, the Italian Panoramic Monochromator, has been built in Arcetri in collaboration with the Physics Department of "Tor Vergata" University in Rome. It essentially consists of a Fabry-Perot interferometer (FPI), used in telecentric mounting, in series with a Universal Birefringent Filter (UBF) (Beckers 1971, 1972), used as an order sorter.

This instrument represents an evolution of a previous one for mono-dimensional spectroscopy, the SpectroInterferometer, which, for many years, was operative at the Solar Tower in Arcetri (Cavallini et al. 1987).

After a first description of the IPM, which can be found in Bonaccini et al. (1989), hereinafter referred to

Send offprint requests to: F. Cavallini e-mail: fabio@utorre.arcetri.astro.it as Paper I, some different versions of this instrument have been built and used in Arcetri for full disk observations, at low spatial resolution (Cavallini et al. 1992). We learnt from this experience that the instrument encounters some severe difficulties in its construction, essentially concerning the correct shape of the overall instrumental profile, its wavelength stability and the spectral homogeneity of the image plane.

As it will be discussed later, these problems have been solved, and when Italy agreed to the THEMIS project in 1992, an improved version of this instrument, for high spatial resolution, has been designed and built. The IPM is operative now and as from April 1997 it is at the disposal of all observers.

\section{The instrumental profile}

\subsection{Parasitic light}

As is well known, the transmission of light through a Fabry-Perot interferometer is given by the Airy function:

$$
T(\lambda)=\left[1+4 R(1-R)^{-2} \sin ^{2}(2 \pi \mu t \cos \theta / \lambda)\right]^{-1}
$$

where $R$ is the coating reflectance, $t$ the spacing between the plates, $\mu$ the refractive index of the material between the plates (air in our case), $\theta$ the angle of incidence, and $\lambda$ the wavelength of the incident light.

For fixed $\mu, t$ and $\theta$, the transmitted light consists of a series of narrow peaks (orders), the separation of which in wavelength is the Free Spectral Range (FSR), and for small $\theta$ :

$\mathrm{FSR}=\lambda^{2} / 2 \mu t$

Let us suppose that the interferometer plates are perfectly flat and parallel ( $t$ constant), and that the interferometer is illuminated by a perfectly collimated raybundle, normally incident on the plates $(\theta=0)$. In this case, the Full Width at Half Maximum (FWHM) of each order of the FPI only depends on the reflectance, and with sufficient accuracy, for $R$ greater than about 0.85 , it is:

$\delta \lambda_{\mathrm{R}}=\lambda^{2}(1-R) / 2 \pi \mu t \sqrt{R}$. 
Due to the periodicity of its passband, an FPI obviously demands a prefilter to be used as a spectroscopic device. In the case of an emission spectrum, with well spaced lines, an interference filter may be sufficient, but in the case of an absorption spectrum, like the solar one, a single order of the interferometer must be isolated. For this reason, the passband of the order sorter must be narrower than the interferometer FSR, which in turn, through Eqs. (2) and (3), depends on the required FWHM of the transmission order. If the use of the FPI is not limited to the strongest solar lines, the isolated order must be significatively narrower than a typical photospheric line, with a FWHM $\simeq$ $100-200 \mathrm{~m} \AA$. If we impose $\delta \lambda \simeq 20 \mathrm{~m} \AA$, corresponding to a spectral resolution $\mathcal{R} \equiv \lambda / \delta \lambda \simeq 300000$, a FSR $\simeq 1 \AA$ is obtained for the interferometer. An order sorter with a FWHM $\leq 1 \AA$ will be therefore necessary.

As described in Paper I, on the IPM a Universal Birefringent Filter is used as order sorter for the FPI. Each passband of the UBF has a shape which resembles a sinc ${ }^{2}$ function, with a FWHM $=0.250 \AA$ and a FSR $=128 \AA$ at $\mathrm{H}_{\alpha}$. An interference filter $(\mathrm{IF})$, with a $\mathrm{FWHM} \simeq 50 \AA$, is then sufficient to isolate a single UBF passband.

From the spectroscopic point of view, the instrument therefore consists of three filters mounted in series. If $W_{\mathrm{IF}}(\lambda), W_{\mathrm{UBF}}(\lambda)$ and $W_{\mathrm{FPI}}(\lambda)$ respectively are the IF, UBF and FPI spectral transmissions, the overall instrumental profile $W(\lambda)$ is:

$W(\lambda)=W_{\mathrm{IF}}(\lambda) W_{\mathrm{UBF}}(\lambda) W_{\mathrm{FPI}}(\lambda)$.

When the maximum of the UBF transmission profile coincides with one of the FPI orders, it follows that the overall instrumental profile consists of one main peak and some side lobes. These unwanted transmission bands contribute to the parasitic light, defined as:

$P=\int_{\lambda_{1}}^{\infty} W(\lambda) \mathrm{d} \lambda / \int_{\lambda_{0}}^{\lambda_{1}} W(\lambda) \mathrm{d} \lambda$

where $\lambda_{0}$ and $\lambda_{1}$ respectively are the wavelength of the transmission peak and of the first zero of the UBF passband.

As shown in Paper I, the parasitic light can be minimized by a suitable matching of the interferometer FSR with the spacing of the UBF zeroes. However, it must be taken into account that, decreasing the operating wavelength, the relative wavelength position of the UBF passband zeroes shrinks with respect to the maxima of the FPI transmission orders. For extended operating wavelength ranges, this therefore prevents the minimization of the parasitic light contribution from the UBF side lobes, that is obtained by adjusting the adjacent orders of the FPI on the first zeroes of the UBF passband. A realistic compromise is obtained by choosing an interferometer with a $3 \mathrm{~mm}$ plate separation. In this case, the side orders of the FPI coincide with the third zeroes of the UBF passband at $\mathrm{H}_{\alpha}(\mathrm{FSR}=0.75 \AA)$. The parasitic light is minimum $(\simeq 0.7 \%)$ at $6563 \AA$, and maximum $(\simeq 2.2 \%)$ at the blue edge of the useful wavelength range (4600 $\AA$ ).

In practice, however, the aforesaid suppression of the unwanted orders of the interferometer is not secured if the UBF passband sensibly differs from the theoretical one and/or the tuning between the UBF and the FPI is not correct. These conditions therefore require a very careful research of the tune solutions for the nine crystal groups forming the UBF (see Paper I), and a wavelength stability of the UBF and of the FPI such as to secure variations of the parasitic light and of the overall passband transparency smaller than the required photometric precision $(1 \%)$.

For a relative detuning of $9 \mathrm{~m} \AA$ at $5500 \AA$, the peak transparency of the instrumental profile decreases of $1 \%$, while the parasitic light increases of $0.4 \%$. As the interferometer is very stable in wavelength (see Sect. 2.3), this result requires a wavelength stability of the UBF better than about $10 \mathrm{~m} \AA$. This is obtained by measuring the resistance of a platinum wire, wound on the crystal groups, and by applying small corrections to the angular position of each group. The drifts of the UBF passband can then be compensated, securing a wavelength stability of $\pm 1 \mathrm{~m} \AA$, better than required.

\subsection{Spectral resolution}

As shown in Sect. 2.1, to reduce the parasitic light, a maximum plate separation of $3 \mathrm{~mm}$ must be adopted for the interferometer. This condition imposes a lower limit to the FWHM of the instrumental profile (Eq. (3)), lowering therefore the attainable spectral resolution. In particular, for a coating reflectance $R=0.95$ (see Table 1 ):

$$
\delta \lambda_{\mathrm{R}}=8.2 \mathrm{~m} \AA \text { and } \mathcal{R}_{\mathrm{R}}=670000 \text { at } 5500 \AA .
$$

Although the reflective resolution is much larger than the demanded one $(\mathcal{R} \simeq 300000)$, we have to consider that in practice, if the raybundle associated with a given image point contains a range of $\theta$ values and/or covers an area of the FPI plates where, due to flatness errors, the plate separation $t$ fluctuates, the transmission profile will be broadened with respect to the reflective one (see later). To evaluate the effective spectral resolution, we have therefore to consider the geometry of the rays incident on the interferometer plates.

Two different optical mountings can be adopted. In the case of the so called classical mounting, the image formed by the telescope is collimated and the FPI is placed near the image of the entrance pupil. Each image point is then formed by a camera lens, which focuses a beam of rays propagating through the interferometer at the same angle with respect to the optical axis. In this case, the FWHM of each order of the FPI is larger than the reflective one $\left(\delta \lambda_{\mathrm{cl}}>\delta \lambda_{\mathrm{R}}\right)$. In fact, the raybundle associated with each image point covers an area of the FPI plates within which 
the plate separation $\mathrm{t}$ fluctuates; for $\theta=0$, this is accounted for by the $2^{\text {nd }}$ term in the following equation:

$$
\begin{aligned}
\delta \lambda_{\mathrm{cl}} & =\left[\delta \lambda_{\mathrm{R}}^{2}+\delta \lambda_{\mathrm{D}}^{2}\right]^{1 / 2} \\
& =\left[\left(\lambda^{2}(1-R) / 2 \pi \mu t \sqrt{R}\right)^{2}+\left(\lambda^{2} / p t\right)^{2}\right]^{1 / 2}
\end{aligned}
$$

where $p$ are the flatness errors (defects) in fractions of $\lambda$.

Moreover, for what concerns the off-axis image points, the largest effect is a shift of the transmission orders towards the blue $\left(\Delta \lambda_{\theta}\right)$, following the well known equation:

$\Delta \lambda_{\theta}=-\lambda \theta^{2} / 2$

In classical mounting, therefore, the final image plane is spectrally dishomogeneous, in the sense that the wavelength of each order is not the same on each point, but depends on its distance from the optical axis, and this is also true for a perfect interferometer.

Instead, in the case of the so called telecentric mounting, the entrance pupil is collimated and the FPI is placed near an image plane. Consequently, all the image points are formed by ray cones normally incident on the interferometer and containing all the possible directions allowed by the optics. In this case, the FWHM of each order of the FPI is larger than the reflective one, because the raybundle corresponding to each image point contains a range of $\theta$ values. Moreover, as the plate separation is not infinitely small, the ray cones always cover a small, but finite area of the FPI plates, and therefore, on each point of the final image plane, the FWHM of each order is also broadened by small scale flatness fluctuations.

In practice, to avoid that the interferometer plates are focused on the final image, the FPI is not placed on the image plane, but as far away as possible from it. In this case, each ray cone corresponding to each image point covers an area of the interferometer plates, the size of which depends on the distance of the FPI from the image and on the relative aperture of the beam. As the beam incident on the interferometer has a very small aperture (see later), the area covered by each ray cone is only some millimeter in size.

In conclusion, the FWHM $\left(\delta \lambda_{\mathrm{tc}}\right)$ of the interferometer orders will be broadened by the flatness errors as well as by the varying $\theta$, accounted for by the $2^{\text {nd }}$ and $3^{\text {th }}$ terms respectively on the following equation:

$$
\begin{aligned}
\delta \lambda_{\mathrm{tc}}= & {\left[\delta \lambda_{\mathrm{R}}^{2}+\delta \lambda_{\mathrm{D}}^{2}+\delta \lambda_{\theta}^{2}\right]^{1 / 2} } \\
= & {\left[\left(\lambda^{2}(1-R) / 2 \pi \mu t \sqrt{R}\right)^{2}+\left(\lambda^{2} / \bar{p} t\right)^{2}\right.} \\
& \left.+\left(\lambda \theta^{2} / 8 \mu\right)^{2}\right]^{1 / 2}
\end{aligned}
$$

where $\bar{p}$ are the small scale flatness errors in fractions of $\lambda$.

Generally $p$ is defined as twice the maximum deviation of the considered surface from its ideal mathematical reference surface; this parameter combines therefore coarse and fine shape errors. However, in telecentric mounting, the broadening of the instrumental profile on each point of the final focal plane is produced by flatness errors, the scale of which is significatively smaller than the area of the interferometer plates. For this reason in Eq. (9) instead of $p, \bar{p}$ has been used, which, as previously said, just represents the surface fluctuations at small scale. Since obviously $\bar{p} \geq p$, the $2^{\text {nd }}$ term in $\delta \lambda_{\mathrm{tc}}$ is generally smaller than the corresponding one in $\delta \lambda_{\mathrm{cl}}$; in other words, the broadening of the instrumental profile produced by flatness errors is generally larger in classical than in telecentric mounting.

Also in the case of telecentric mounting, the final image plane is spectrally dishomogeneous, but in the sense that the wavelength position and the FWHM of each order randomly changes from a point to an other, due to the $t$ fluctuations. However, unlike the case of the classic mounting, this is true only for a real interferometer.

Between the two optical mountings, the telecentric one has been adopted for the IPM. In this case it is possible to minimize the spectral dishomogeneities of the final image plane and to contain the broadening of the instrumental profile, produced by the varying $\theta$, within the limit imposed by the demanded spectral resolution. As a matter of fact, for the adopted interferometer (see Table 1), a surface accuracy of $\lambda / 150$ after coating at $5461 \AA$ is claimed by the manufacturer. As a consequence, the maximum wavelength fluctuation of the instrumental profile on the final image plane is $13.3 \mathrm{~m} \AA$ peak to peak $\left(728 \mathrm{~ms}^{-1}\right)$ at $5500 \AA$. Moreover, assuming $\bar{p}=p=150$, $t=3 \mathrm{~mm}, R=0.95$, and imposing a spectral resolution $\mathcal{R}_{\mathrm{tc}}=300000$ at $5500 \AA$, we obtain from Eq. (9) $\theta=18^{\prime}$, i.e. an $\mathrm{f} / 190$ relative aperture of the beam incident on the interferometer. In classic mounting, instead, an exceedingly small relative aperture (f/820) should be used to have the same wavelength variation from the center to the edge of the field (Eq. (8)). In practice, an $\mathrm{f} / 192$ relative aperture has been adopted, corresponding to $\theta=17.9^{\prime}$.

Assuming $\lambda=5500 \AA, \mu=1, \theta=17.9^{\prime}, R=0.95$, $t=3 \mathrm{~mm}, \bar{p}=p=150$ (see Table 1 ), we find:

$\delta \lambda_{\mathrm{R}}=8.2 \mathrm{~m} \AA, \quad \delta \lambda_{\mathrm{D}} \leq 6.7 \mathrm{~m} \AA, \quad \delta \lambda_{\theta}=18.6 \mathrm{~m} \AA$

where the inequality symbol is due to the assumption $\bar{p}=$ $p$.

Following Eq. (9), we may conclude therefore that the FWHM of the instrumental profile and the spectral resolution of the IPM are:

$\begin{aligned} \delta \lambda_{\mathrm{IPM}} & \leq 21.5 \mathrm{m \AA} \text { and } \\ \mathcal{R}_{\mathrm{IPM}} & =\lambda / \delta \lambda_{\mathrm{IPM}} \geq 256000 \text { at } 5500 \AA .\end{aligned}$

\subsection{Wavelength stability}

As the overall instrumental profile essentially is one order of the interferometer, its wavelength stability essentially depends on the interferometer itself, and, in particular, on the stability of the optical length of the cavity, i.e. the 
Table 1. Fabry-Perot interferometer characteristics

\begin{tabular}{|c|c|}
\hline Manufacturer & Queensgate Instruments L \\
\hline Type & $\begin{array}{l}\text { Mod. ET50 } \\
\text { piezo-scanned and } \\
\text { capacity servo-controlled }\end{array}$ \\
\hline Clear aperture & $50 \mathrm{~mm}$ \\
\hline Plate separation & $3 \mathrm{~mm}$ \\
\hline Wedge angle & $15^{\prime}$ \\
\hline Coating & Multilayer broadband \\
\hline Wavelength range & $4000-7000 \AA$ \\
\hline Reflectivity & 0.95 at $5500 \AA$ \\
\hline $\begin{array}{l}\text { Flatness errors of } \\
\text { each plate at } 5461 \AA\end{array}$ & $\begin{array}{l}\lambda / 150 \text { maximum } \\
\text { after coating }\end{array}$ \\
\hline Cavity tuning & $2000 \mathrm{~nm}$ \\
\hline Resolution & 12 bits \\
\hline $\begin{array}{l}\text { Settling time } \\
\text { (response to step input) }\end{array}$ & $\simeq 1.5 \mathrm{~ms}$ \\
\hline $\begin{array}{l}\text { Temperature sensitivity } \\
\text { of the interferometer }\end{array}$ & $3 \mathrm{~m} \AA{ }^{\circ} \mathrm{C}^{-1}$ at $6438 \AA$ \\
\hline $\begin{array}{l}\text { Temperature sensitivity } \\
\text { of the CS100 controller }\end{array}$ & $0.1 \mathrm{~m} \AA{ }^{\circ} \mathrm{C}^{-1}$ at $6438 \AA$ \\
\hline $\begin{array}{l}\text { Electronic noise equivalent } \\
\text { displacement of interferometer } \\
\text { plates with CS100 }\end{array}$ & $10 \mathrm{pm} \mathrm{Hz}^{-1 / 2} \mathrm{rms}$ \\
\hline
\end{tabular}

product between the geometrical distance of the plates and the refractive index of the air between them.

On the adopted interferometer, the plate separation, as well their parallelism, are stabilized by a capacitance servo-control. A three channel bridge system, the CS100, uses capacitance micrometers to derive error signals when the spacing or the parallelism change. These signals are then used to drive piezo-electric staks which move to correct the errors. Because this is a close-loop system, nonlinearity and hysteresis in the piezo-electric are entirely removed.

The CS100 however can erroneously adjust the geometrical distance of the plates, thus producing wavelength instabilities of the interferometer, due to different effects: i) changes in the dielectric constant of the air, producing a wrong signal from the capacitance micrometers, ii) changes in the spacing between the pads of the micrometers, produced by thermal effects on the capacitor pillars, iii) electronic drifts produced by thermal effects on some components of the CS100.
Moreover, for what concerns the refractive index of the air, this obviously depends on the atmospheric changes of pressure, temperature and humidity.

The environmental effects on both the capacitance micrometers and the optical length of the cavity are easily eliminated by placing the interferometer in a sealed chamber. The residual thermal effects have then been eliminated by accurately thermostatizing both the interferometer and the electronics.

In particular, as the FPI has a thermal sensitivity of $3 \mathrm{~mA}{ }^{\circ} \mathrm{C}^{-1}$, to obtain a wavelength stability of $\pm 1 \mathrm{~ms}^{-1}$, the interferometer must be thermostatized within \pm 5 $10^{-3}{ }^{\circ} \mathrm{C}$. To this purpose the FPI chamber has been enclosed in a copper box, externally warmed by a sheet resistance, and covered by heat insulating panels; two fans mix the air to make the temperature uniform inside the box, and a thermostatic system allows one to obtain a thermal stability of $\pm 0.1{ }^{\circ} \mathrm{C}$ at about $37^{\circ} \mathrm{C}$. Then, a second sheet resistance, wrapped around the sealed chamber and controlled by a second independent thermostatic system, maintains the interferometer at about $38^{\circ} \mathrm{C}$ within $\pm 510^{-3}{ }^{\circ} \mathrm{C}$ (Fig. 1), for variations of $\pm 2{ }^{\circ} \mathrm{C}$ of the ambient temperature.

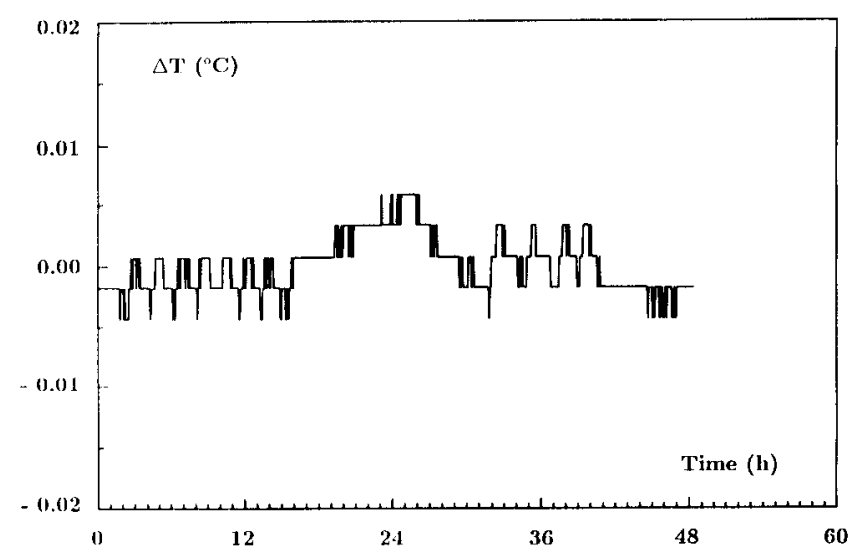

Fig. 1. Temperature inside the interferometer chamber vs. time. The temperature has been evaluated by measuring the resistance of a Pt100, with a resolution of $1 \mathrm{~m} \Omega( \pm 2.5$ $\left.10^{-3}{ }^{\circ} \mathrm{C}\right)$, and the fluctuations relative to the mean $\left(\simeq 38{ }^{\circ} \mathrm{C}\right)$ have been plotted

As regards the $\mathrm{CS} 100$, its thermal sensitivity is $0.1 \mathrm{~m} \AA{ }^{\circ} \mathrm{C}^{-1}$, and a thermostatization within $\pm 0.1{ }^{\circ} \mathrm{C}$, obtained by controlling the speed of two fans cooling the electronics, is therefore sufficient to secure the demanded wavelength stability.

Finally, to test the residual drift of the interferometer, the ${ }^{114} \mathrm{Cd}$ red line at $6438 \AA$, emitted by an isotopic spectral lamp, has been used. The stability of this line (secondary wavelength standard) is $\pm 210^{-8} \lambda$, corresponding to $\pm 6 \mathrm{~ms}^{-1}$ in velocity units (Engelhard \& Bayer-Helms 1972). 
The following assembly has then been used to perform the test. The interferometer, used in axial mode, was placed between two lenses: the first one collimates a pinhole, where a macrophoto lens forms an image of the lamp discharge tube, the second one forms an image of the pinhole on the cathode of a photomultiplier. A second photomultiplier, used as reference, directly sees, via a fiber optic, the light emitted by the lamp. The photomultipliers are both used in photon counting and are preceded by an interference filter, centered at $6438 \AA$, sufficiently narrow (FWHM $=50 \AA$ ) to isolate the Cd red line.

This assembly was first used to accurately measure the profile of the $\mathrm{Cd}$ line and to locate on it a linear range, which was found between 60 and $80 \%$ of the peak intensity. The two points, at the center of the linear ranges on the blue and on the red flank of the line, have been then located at $\pm 13 \mathrm{~m} \AA$ from the line peak.

Let us define now as velocity signal the quantity:

$S\left(\Lambda-\lambda_{0}\right)=\left(I_{\mathrm{B}} / R_{\mathrm{B}}-I_{\mathrm{R}} / R_{\mathrm{R}}\right) /\left(I_{\mathrm{B}} / R_{\mathrm{B}}+I_{\mathrm{R}} / R_{\mathrm{R}}\right)$

with

$\Lambda=\left(\lambda_{\mathrm{B}}+\lambda_{\mathrm{R}}\right) / 2$

where $\lambda_{0}, \lambda_{\mathrm{B}}$ and $\lambda_{\mathrm{R}}$ respectively are the wavelengths of the line peak and of two points on the blue and on the red flank of the line; $I_{\mathrm{B}}$ and $I_{\mathrm{R}}$ are the intensities measured on $\lambda_{\mathrm{B}}$ and $\lambda_{\mathrm{R}} ; R_{\mathrm{B}}$ and $R_{\mathrm{R}}$ are the corresponding intensities measured by the reference photomultiplier. Fixed $\lambda_{\mathrm{R}}-$ $\lambda_{\mathrm{B}}=26 \mathrm{~m} \AA$, we have, within the linear range:

$S\left(\Lambda-\lambda_{0}\right)=K \times\left(\Lambda-\lambda_{0}\right)$

$K$ has been then evaluated by a linear fitting of $S$, measured at five $\Lambda$ around $\lambda_{0}(\Delta \Lambda=1 \mathrm{~m} \AA)$. The so obtained value for $K$ is:

$K=0.065 \mathrm{~m} \AA^{-1}$.

It follows from Eqs. (13) and (14) that, if the wavelength stability of the interferometer must be measured within $\pm 1 \mathrm{~ms}^{-1}( \pm 0.02 \mathrm{m \AA})$, we must be able to measure a variation $\Delta S= \pm 1.310^{-3}$. It is easy to show that, assuming $I_{\mathrm{B}} \simeq I_{\mathrm{R}} \simeq R_{\mathrm{B}} \simeq R_{\mathrm{R}} \equiv I$, the rms errors on $S$ and $\Delta S$ respectively are $\sigma_{\mathrm{S}}= \pm(1 / I)^{1 / 2}$ and $\sigma_{\Delta \mathrm{S}}= \pm(2 / I)^{1 / 2}$. If we impose $\Delta S \geq 3 \sigma_{\Delta \mathrm{S}}$, we obtain therefore $I \geq$ $10^{7}$ counts.

To measure this signal in a short time, a photon counting at frequencies of some $\mathrm{MHz}$ is obviously necessary. At this rate however the non linearity effects become relevant and the dead time of the chain formed by the photomultiplier, the amplifier-discriminator and the counter must be known. To this purpose, the following second assembly has been used. By means of a beamsplitter cube, the light emitted by a photodiode is seen simultaneously by the two photomultipliers. Between the diode and the cube a shutter allows one to measure the dark counting and a filter wheel, carrying a neutral density $(D=0.1)$, allows one to reduce the signal of a known amount. For each photomultiplier, the ratio $R$ between the signals with and without density has then been measured at different light levels, up to $6.3 \mathrm{MHz}$. $R$ has been then reported versus $\log I$, where $I$ is the signal without density, and the dead time has been obtained by the best fit of the data. The same dead time has been found for the two photomultipliers (38 ns) and, accounting for it, the newly measured $R$ are constant within $0.5 \%$, up to $6.3 \mathrm{MHz}$.

The first described assembly, used to measure the profile of the Cd red line, has been then used to measure the wavelength stability of the interferometer. To this purpose, the position of the line peak has been located, and then the velocity signal $S$ has been measured on two points at $\pm 13 \mathrm{~m} \AA$ from the line peak and on two other points at $\pm 14 \mathrm{~mA}$. By using crossed polarizers, the signals on the two photomultipliers have been adjusted to $\simeq 2 \mathrm{MHz}$ and an integration time of $5 \mathrm{~s}$ has been used, to secure a sensitivity of $1 \mathrm{~ms}^{-1}$. The measurement has been repeated each minute for two days and the results are shown in Fig. 2, where the wavelength drift, obtained by Eqs. (13) and (14), is plotted versus time. The curves $a$ and $b$ respectively refer to the measurements performed at \pm 13 and $\pm 14 \mathrm{~m} \AA$, and, for the sake of clarity, they have been arbitrarily shifted along the $y$ axis. To evaluate the error on the measured drifts, the curve $c$ has been then obtained as a difference between $a$ and $b$. As it may be seen, $c$ is a horizontal straight line with a superimposed noise, amounting to $\pm 0.85 \mathrm{~ms}^{-1}$. If we suppose that the error on $a$ and $b$ is similar, we may conclude that the error on each measured drift is $\pm 0.60 \mathrm{~ms}^{-1}$, significatively larger than the expected error $\sigma_{\Lambda}=\sigma_{\mathrm{S}} \mathrm{K}^{-1}= \pm 4.910^{-3} \mathrm{m \AA}$ $\left( \pm 0.23 \mathrm{~ms}^{-1}\right)$. This discrepancy can be explained as due to the electronic noise of the CS100, which causes the interferometer plates to make small amplitude random movements about their mean position. Queensgate claims an electronic noise equivalent displacement of the FPI plates amounting to $\pm 10 \mathrm{pm} \mathrm{Hz}^{-1 / 2}$ (see Table 1 ). For a $3 \mathrm{~mm}$ separation, this means a noise $\sigma_{\mathrm{CS} 100}=$ $\pm t^{-1 / 2} \mathrm{~ms}^{-1} \mathrm{~Hz}^{-1 / 2}$, where $t$ is the integration time. If $t=5 \mathrm{~s}$, the electronic noise will be therefore $\sigma_{\mathrm{CS} 100}=$ $\pm 0.45 \mathrm{~ms}^{-1}$, and finally the total expected noise, photometric plus electronic, will be $\pm 0.5 \mathrm{~ms}^{-1}$, very similar to the measured value of $\pm 0.6 \mathrm{~ms}^{-1}$.

By carrying on the analysis of Fig. 2, it may be seen that the $a$ and $b$ curves show a very slow, nearly linear trend. From a linear fitting to the mean of the two curves, a drift of $0.014 \mathrm{~mA} \mathrm{~h}^{-1}\left(0.65 \mathrm{~ms}^{-1} \mathrm{~h}^{-1}\right)$ is found. This drift, amounting to $0.67 \mathrm{~m} \AA\left(31 \mathrm{~ms}^{-1}\right)$ in $48 \mathrm{~h}$, cannot be ascribed to the the spectral line, the stability of which is $\pm 6 \mathrm{~ms}^{-1}$, neither to temperature effects on the interferometer, as shown by Fig. 1, but more probably to some electronic drift of the CS100. Other shorter measurements of the interferometer wavelength stability have been performed and different drifts have been found, but 


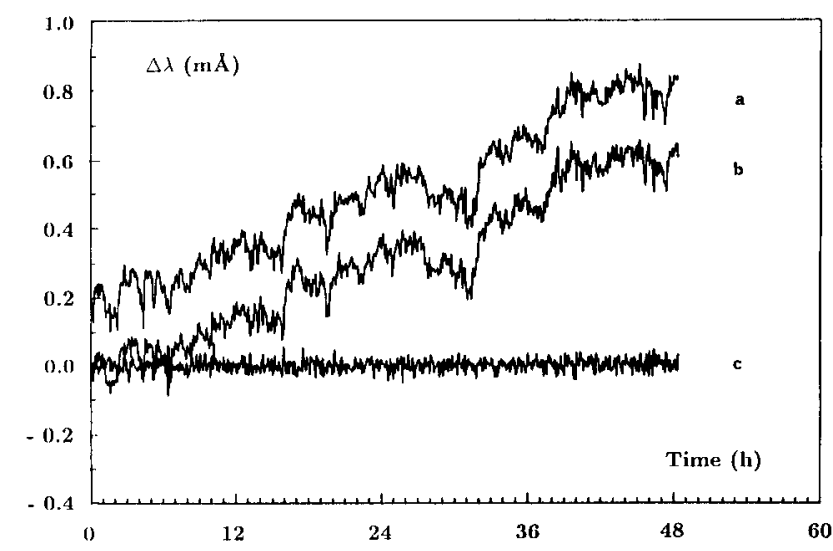

Fig. 2. Wavelength drift of the Fabry-Perot interferometer vs. time. The curves $a$ and $b$ refer to independent measurements of the $6438 \AA \mathrm{Cd}$ line position, respectively at \pm 13 and $\pm 14 \mathrm{~m} \AA$ from the line peak. For the sake of clarity, the two curves have been arbitrarily shifted along the $y$ axis. The curve $c$, a horizontal straight line with a superimposed noise of $\pm 0.85 \mathrm{~ms}^{-1}$, has been obtained as a difference between $a$ and $b$

none larger than $5 \mathrm{~ms}^{-1}$ has been observed over a period of ten hours.

Moreover, the residuals of the linear fitting, shown in Fig. 3, evidentiate wavelength instabilities amounting to $0.34 \mathrm{~m} \AA\left(16 \mathrm{~ms}^{-1}\right)$ peak to peak. The cause of these fluctuations is not evident; their amplitude, however, very similar to the line instability, suggests that probably they must be ascribed to the spectral lamp, rather than to the interferometer itself. To test if these fluctuations could be ascribed to the lamp power supply, the recommended cathod heating current $(0.8 \mathrm{~A})$ and the discharge current $(0.3 \mathrm{~A})$ have been changed from 0.6 to $0.9 \mathrm{~A}$ and from 0.2 to $0.5 \mathrm{~A}$ respectively, but no variation on the velocity signal within $\pm 1 \mathrm{~ms}^{-1}$ has been observed. It is evident, therefore, that to have a more accurate knowledge of the instrumental wavelength drift a more stable source, as, for example, a frequency stabilized laser, should be used.

In conclusion, after the inclusion of the interferometer in a sealed chamber and a careful thermostatization, the instrumental profile shows a very high wavelength stability: the maximum expected drift on $10 \mathrm{~h}$, as in the case of a very long solar observation, is about $0.2 \mathrm{~m} \AA\left(10 \mathrm{~ms}^{-1}\right)$.

Anyhow, as a measurement of the instrumental wavelength drift demands a short time to be performed $(\simeq 2.5 \mathrm{~s}$ for an integration time of $1 \mathrm{~s})$, such a measurement can be alternated to the observations and used to compensate the drift, by correcting the biasing voltage applied to the interferometer. However, in practice, because the smallest voltage step allowed by the CS100 corresponds to a wavelength shift of about $1 \mathrm{~m} \AA$, too large with respect to the observed drift, this correction will seldomly be useful: when the instrument does not work correctly.

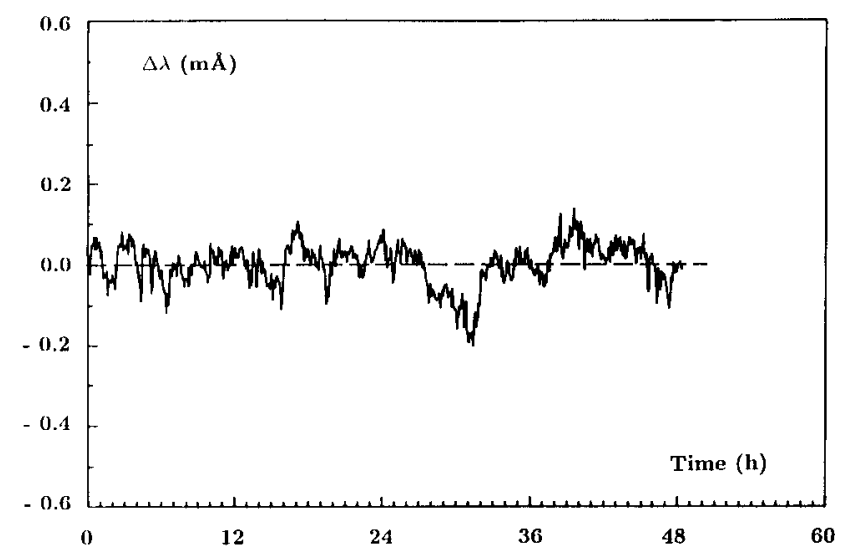

Fig. 3. Residuals of a linear fitting to the mean of the $a$ and $b$ curves of Fig. 2, showing wavelength instabilities amounting to $0.34 \mathrm{~mA}\left(16 \mathrm{~ms}^{-1}\right)$ peak to peak

On the other hand, the measurement of the drift can be also useful a posteriori to verify the instrumental behaviour, and, in case, to correct the data for drifts exceeding the wavelength stability of the Cd lamp.

\section{The instrument}

\subsection{The telescope}

The THEMIS telescope is an alto-azimuthal mounting where all the post-focus instrumentation is contained in a cylindrical tank rotating with the telescope around a vertical axis. The primary mirror is $0.9 \mathrm{~m}$ in diameter with an equivalent focal length of $54.861 \mathrm{~m}$ (f/61). A transfer optics forms an image, $\simeq 4^{\prime}$ in diameter, on a secondary focus (F2), where the scale is $0.266 \mathrm{~mm} \operatorname{arcsec}^{-1}$ and the FWHM of the point spread function, for diffraction limited optics, is $0.15^{\prime \prime}$ at $5500 \AA$.

When it was decided to install the IPM on THEMIS, the telescope was already completely designed and partially built in Orsay (Paris). Many mechanical constraints, therefore, have been imposed to the instrumental project, as: i) a $90^{\circ}$ folding (M2 in Fig. 4) at $610 \mathrm{~mm}$ from F2, breaking the instrument in a horizontal and a vertical path, ii) a maximum volume for the vertical path of $500 \mathrm{~mm} \times 500 \mathrm{~mm} \times 3100 \mathrm{~mm}(\mathrm{~W} \times \mathrm{P} \times \mathrm{H})$, iii $) \mathrm{a}$ $300 \mathrm{~mm}$ avoidance zone for access to the spectrograph at $1650 \mathrm{~mm}$ from M2, iv) a reduction of the available volume on the last $400 \mathrm{~mm}$, for a second access to the spectrograph.

The IPM was therefore mounted, in part to a horizontal rail jutting out from the tank, in part to vertical rails fixed on the external wall. A moveable mirror, just before F2, horizontally folds the sunlight coming from the telescope towards the IPM. This mirror (M1 in Fig. 4) can be replaced by a dichroic, allowing to carry out, in different wavelength ranges, simultaneous observations with the IPM and the French spectrographs. 


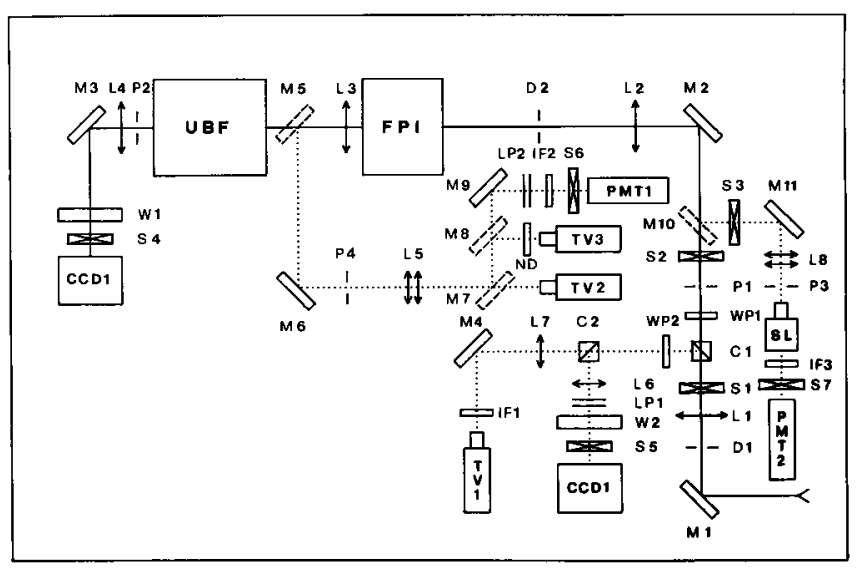

Fig. 4. Schematic drawing of the instrumental layout. The solid line represents the principal optical path, while the secondary ones are showed by dotted lines. Dashed boxes represent moveable mirrors, which can be pulled in or out the optical path. The meaning of the labels is as follows. C: polarizing beamsplitter cube, CCD: CCD camera, D: diaphragm, FPI: Fabry-Perot interferometer, IF: interference filter, L: lens, LP: linear polarizers, M: mirror, ND: neutral density, P: pinhole, PMT: photomultiplier, S: electronic shutter, SL: spectral lamp, TV: TV camera, UBF: Universal Birefringent Filter, W: filter wheel, WP: wave plate

\subsection{The principal optical path}

The principal optical path is shown by the solid line in Fig. 4, schematically representing the instrumental layout.

The image is formed by the telescope on the field diaphragm D1, $13.5 \mathrm{~mm}$ in diameter, corresponding to $51^{\prime \prime}$. An optical target can be mounted on this diaphragm, to allow the positioning of the two CCD cameras on the focus and on the same field of view. Moreover, two perpendicular tongues on D1 can be used as geometrical references, to superimpose a posteriori the monochromatic and the reference images taken by CCD1 and CCD2. The whole acquisition system will be described in a separate paper; a preliminary description is already available (Cantarano et al. 1993).

The image on D1 is then collimated by a first lens L1, followed by the shutter S1, allowing to optically isolate the IPM from the telescope, and by the achromatic polarizing beamsplitter cube $\mathrm{C} 1$, sending one half of the incoming radiation to TV1 and CCD2. The cube $\mathrm{C} 1$ is then followed by the achromatic half wave plate WP1, which rotates the transmitted $\mathrm{p}$ polarized radiation to align its direction with that of the linear polarizer at the entrance of the UBF. Because the UBF has a fixed linear polarizer at the exit, to better exploit the incoming radiation, one half of it, that would remain unused, is folded at the entrance by $\mathrm{C} 1$ and then the UBF, which cannot work in linearly polarized light, is used back to front.

Going on along the principal optical path, we find the pinhole P1, which, by isolating the image of the entrance pupil formed by L1, reduces the instrumental stray light, then the shutter S2, used to separate the principal optical path from that of the spectral lamp SL, and then the mirror M2, folding the radiation in the vertical part of the instrument.

A second lens L2 then collimates the pupil, forming on the diaphragm D2 an enlarged image of the solar region selected by D1. Two perpendicular tongues on D2, opposite to those on D1, can be used as geometrical references to superimpose the monochromatic images to the spectral dishomogeneities maps, obtained by means of the Cd spectral lamp (see Sect. 4).

After D2, in an image space where the pupil is collimated, we find the interferometer, used in axial mode and in telecentric mounting.

A third lens L3, just after the interferometer, then collimates the image, forming beyond the UBF a new image of the pupil on the pinhole $\mathrm{P} 2$, used to remove the ghost images produced by reflections on the rear surfaces of the interferometer plates. To this purpose, the plates are wedged by $\alpha=15^{\prime}$, and as the minimum deflection is $2 \alpha$, for a relative aperture smaller than $\mathrm{f} / 115$, all the spurious images are separated and can be easily removed by a suitable pinhole, as P2, used to isolate the on-axis pupil image.

Finally, a forth lens L4 and a third folding mirror M3 form a monochromatic image on the CCD1 camera. This camera is preceded by the shutter S4, controlling the exposure time, and by a filter wheel $\mathrm{W} 1$, carrying the interference filters for the wavelength ranges where the UBF is calibrated (see Table 2).

The CCD is a Thompson $9.7 \mathrm{~mm} \times 9.7 \mathrm{~mm}$, with $512 \times 512$ square pixels, $19 \mu$ in size. Obviously, the image scale on the CCD must be such as not to reduce the telescope spatial resolution. As the overall field is $51^{\prime \prime}$ and the FWHM of the point spread function is $0.15^{\prime \prime}$, the maximum number of resolved elements is 340. Assuming therefore two pixels per resolved element, and an useful square field inscribed in the total admitted circular field, an at least $481 \times 481$ pixels detector and an image scale of $0.077^{\prime \prime}$ pixel $^{-1}$ or smaller are required. In our case, we have an useful field of $33^{\prime \prime} \times 33^{\prime \prime}$ with an image scale of $0.065^{\prime \prime}$ pixel $^{-1}$, allowing therefore to exploit all the telescope spatial resolution.

\subsection{The reference path}

This secondary path originates from the achromatic polarizing beamsplitter cube $\mathrm{C} 1$. The reflected s polarized light passes through WP2, an achromatic half wave plate, which can be rotated to differently distribute the light between CCD2 and TV1 by means of the polarizing cube C2. The lens L6 forms on CCD2 an image of the same solar region seen by CCD1. LP1 are two crossed linear polarizers, used to adjust the light level, W2 is a filter wheel, identical to $\mathrm{W} 1$, and finally $\mathrm{S} 5$ is a shutter, controlling 
Table 2. The Italian Panoramic Monochromator characteristics

\begin{tabular}{|c|c|}
\hline Wavelength range & $4600 \AA-6800 \AA$ \\
\hline Calibrated ranges & $\begin{array}{l}5184 \AA(\mathrm{Mg} \mathrm{b} 1), 5380 \AA(\mathrm{C} \mathrm{I}), \\
5576 \AA(\text { Fe I }), 5890 \AA(\mathrm{Na} \mathrm{D} 2), \\
6438 \AA \text { (Cd red line), } 6563 \AA(\mathrm{H} \\
\pm 20 \AA\end{array}$ \\
\hline Spectral resolving power & $\begin{array}{l}\geq 256000(\mathrm{FWHM} \leq 21.5 \mathrm{m \AA}) \\
\text { at } 5500 \AA\end{array}$ \\
\hline Wavelength drift & $\leq 10 \mathrm{~ms}^{-1}$ on $10 \mathrm{~h}$ \\
\hline $\begin{array}{l}\text { Spectral homogeneity } \\
\text { (after correction) }\end{array}$ & $\pm 10 \mathrm{~ms}^{-1} \mathrm{rms}$ \\
\hline Field of view & $51 "$ \\
\hline Detectors & $\begin{array}{l}\text { Thomson CCDs } \\
512 \times 512 \text { square pixels } \\
19 \mu \mathrm{m} \text { in size; } \\
9.7 \mathrm{~mm} \times 9.7 \mathrm{~mm} \Rightarrow 33^{\prime \prime} \times 33^{\prime \prime}\end{array}$ \\
\hline & $\begin{array}{l}\text { A/D converter: } 12 \text { bit } \\
\text { Full-frame read time: } 2.6 \mathrm{~s} \\
\text { Read noise: } 30 \mathrm{e}^{-}\end{array}$ \\
\hline Image scale & $\begin{array}{l}0.065^{\prime \prime} \text { pixel }^{-1} \\
(\simeq 2 \text { pixels } / \text { resolved element })\end{array}$ \\
\hline Wavelength setting time & $\begin{array}{l}\leq 1 \mathrm{~s} \\
(\text { typically } \simeq 0.1 \mathrm{~s})\end{array}$ \\
\hline $\begin{array}{l}\text { Exposure time ( } 1 \% \text { photometric } \\
\text { precision on the continuum) }\end{array}$ & $\simeq 0.1 \mathrm{~s}$ \\
\hline $\begin{array}{l}\text { Acquisition rate including: } \\
\text { wavelength setting, exposure, } \\
\text { frame reading, display and storing }\end{array}$ & $\simeq 0.2$ frames $\mathrm{s}^{-1}$ \\
\hline
\end{tabular}

the exposure time. The CCD2 camera allows to obtain, simultaneously with CCD1, images of the same solar area on the same spectral region, but with a larger passband ( $\simeq 50 \AA)$. These white light images can be then used as reference and/or for destretching procedures, to correct a posteriori distortion and image motion produced by seeing.

The lens L7 then forms an image of all the admitted circular field selected by D1 on the TV camera TV1, preceded by the mirror M4, the interference filter IF1 $(\lambda=$ $5300 \AA, \mathrm{FWHM}=50 \AA$ ) to increase the contrast, and a macrophoto lens. TV1, continuously showing the selected solar region, can be used to monitor the solar and atmospheric conditions.

\subsection{The path of the spectral lamp}

The spectral lamp SL is the same already described in Sect. 2.3. A macrophoto lens forms an 1:1 image of the lamp discharge tube on the pinhole P3, with the same diameter of the image of the entrance pupil, formed by L1. The two lenses L8 and the mirror M11 form then an 1:1 image of the pinhole, the position of which coincides with that of the entrance pupil, when the moveable mirror M10 is inserted on the principal optical path. In this case the FPI and the UBF see the lamp in the same way as the Sun. A second moveable mirror M5, which can be inserted on the optical path after L3, and the mirror M6 form on the pinhole $\mathrm{P} 4$ an enlarged image of $\mathrm{P} 3$. P4, as $\mathrm{P} 2$, removes the ghost images produced by reflections on the rear surfaces of the interferometer. The two lenses L5 form then: i) an 1:1 image of the pupil on the cathode of the photomultiplier PMT1, by M9 and the moveable mirror M7, ii) the same image in front of the color TV camera TV3, by the two moveable mirrors M7 and M8, iii) an image of the interferometer plates in front of the color TV camera TV2.

PMT1 is preceded by the two crossed linear polarizers LP2, to adjust the light level, by the interference filter IF2 $(\lambda=6438 \AA, \mathrm{FWHM}=50 \AA)$ to isolate the Cd red line, and by the shutter S6. PMT1, which permits the measurement of the wavelength drift of the interferometer, is used together with the reference photomultiplier PMT2, which, via a fiber optic, sees the $\mathrm{Cd}$ line through the interference filter IF3, equal to IF2.

The TV3 camera can be used to verify and, if necessary, to adjust the orthogonality between the optical path and the interferometer plates. To this purpose, TV3 can show the entrance or the spectral lamp pupil, by pulling the moveable mirror M10 in or out the optical path. To compensate the much different luminosity of the two images, the neutral density ND is automatically inserted in front of the macrophoto lens, mounted on TV3, when the entrance pupil is shown. A double cross hair generator allows to verify on a TV monitor the relative position of the two pupil images, and by acting on the mirror M11, it is possible to move the lamp pupil until the two images coincide. When this condition is verified, if the interferometer plates and the optical path are not orthogonal, the image of the lamp pupil, showing the inner part of the ring pattern produced by the FPI, will appear asymmetrical. In this case, by changing the orientation of the interferometer, a symmetrical image can be obtained, securing therefore the correct orthogonality.

The TV2 camera can be used to verify and, if necessary, to adjust the parallelism of the interferometer plates. To this purpose, this camera can show the FPI plates lighted by the spectral lamp radiation. For a perfect interferometer, used in telecentric mounting, the passband wavelength position should be identical on each point of the plates, the image of which, therefore, should appear 
homogeneous. In practice, however, in different points of the plates the interference orders will be randomly and sistematically shifted, due to flatness and parallelism errors. For this reason, the plates will appear dishomogeneous in intensity and sometimes in color, if in different points different lines emitted by the lamp coincide in wavelength with some interferometer order. By observing the plate image shown by TV2 and changing the parallelism conditions up to obtain an image as homogeneous as possible, it is easy to secure the plate parallelism with a precision better than $\lambda / 1000$.

\subsection{Remote controls}

All the procedures which need to adjust the instrument and to verify its correct behaviour must be performed from a remote control room. To this purpose, many instrumental components are provided with actuators for a remote positioning, while three TV cameras allow to verify the correctness of the operations. In the following, these remote controls are described.

Mirror M6: it allows to center on the monitor connected to TV3 the image of the entrance pupil with respect to an electronic cross hair.

Mirror M11: it allows to obtain the coincidence between the entrance and the Cd lamp pupil, verified by the TV3 camera (see Sect. 3.4).

Mirror M3 and cube C2: they allow to center the solar image on the two CCD cameras.

Mirror M4: it allows to center on the monitor connected to TV1 the image of the observed solar region.

FPI: the interferometer is provided with two actuators which allow to rotate it around one axis, normal to the plane of Fig. 4, and around a second axis, contained on this plane and normal to the optical path. In this way it is possible to secure the correct orthogonality between the interferometer plates and the optical axis, verified by the TV2 camera (see Sect. 3.4).

CCD1 and CCD2: each one of the two CCD cameras is mounted on a motorized translation stage, allowing to obtain the correct focusing.

\section{Spectral dishomogeneities}

As described in Sect. 2.2, as a consequence of the adopted telecentric mounting, the final focal plane is spectrally dishomogeneous, with wavelength fluctuations of the instrumental profile amounting to $13.3 \mathrm{~m} \AA$ peak to peak $\left(728 \mathrm{~ms}^{-1}\right)$ at $5500 \AA$. The Cd spectral lamp, however, can be used to measure such fluctuations, allowing to account for them a posteriori.

Referring to Fig. 4, when the mirror M10 is on the optical path and M5 is out, the lens L4 forms on CCD1 an image of the diaphragm D2, lighted by the lamp radiation. A series of images taken while the interferometer is scanning the Cd red line allows then to measure on each pixel the line peak position; as reference in this case the PMT2 photomultiplier is used. By evaluating on each pixel the line wavelength shift relative to the mean on the overall field, a map of the spectral dishomogeneities is obtained. A map like this, as a sort of spectral flat field, allows then to correct the solar images for flatness and parallelism errors.

Ten maps have been sequentially obtained, and a mean error of $\pm 0.2 \mathrm{~m} \AA\left( \pm 9 \mathrm{~ms}^{-1}\right)$ in evaluating the wavelength shift has been found. We may conclude therefore that this procedure allows to measure and correct the spectral dishomogeneities of the image plane with a precision better than $\pm 10 \mathrm{~ms}^{-1}$.

The same maps, which can be considered as a representation of the interferometer cavity errors, can be also used to evaluate the surface accuracy. A peak to peak variation of $16 \mathrm{~m} \AA$ on the line position has been found, corresponding to flatness errors of $\lambda / 146$ at $5461 \AA$, very similar to the $\lambda / 150$ value, claimed by the manufacturer.

\section{Other instrumental characteristics}

The more relevant instrumental characteristics are reported in Table 2, and only those up to now not examined will be discussed in this section.

Wavelength range: while for the UBF the useful wavelength range is $4200-7000 \AA$, and for the interferometer $4000-7000 \AA$, the IPM can be used only on the range $4600-6800 \AA$. This limitation is imposed by the achromatic ranges of the wave-plates and of the polarizing beemsplitter cubes.

Calibrated ranges: due to its very complex construction, the UBF shows an optical behaviour which significatively differs from the theoretical one. The calibration procedure, therefore, demands a lot of time to be performed and the use of a spectrograph with a medium-high spectral resolution. For this reason the filter has been calibrated only on six wavelength ranges, $40 \AA$ wide, centered on lines of astrophysical interest. The range at $6438 \AA$ has been calibrated to allow the measurement of the spectral dishomogeneities of the focal plane, but obviously it can be also used for solar observations. In principle the calibration of the UBF can be extended to other ranges, but since in this case the filter should be brought back to Arcetri, such a possibility will be re-examinated only in the future.

Field of view: in telecentric mounting the maximum obtainable angular field $\beta$ linearly depends on the allowed incidence angle $\theta$ (see Paper I):

$\beta=\theta \Phi_{\mathrm{FPI}} / \Phi_{\mathrm{TEL}}$

where $\Phi_{\mathrm{FPI}}$ and $\Phi_{\mathrm{TEL}}$ respectively are the interferometer and the telescope diameters. Assuming $\theta=17.9^{\prime}$ (see Sect. 2.2), $\Phi_{\mathrm{FPI}}=50 \mathrm{~mm}$ and $\Phi_{\mathrm{TEL}}=900 \mathrm{~mm}$, a maximum angular field $\beta=1^{\prime}$ is obtained. In practice, as not all the clear aperture of the FPI can be used, the useful angular field is $\beta=51^{\prime \prime}$, which, as described in Sect. 3.2, 
circumscribes a square CCD: the resulting useful field is therefore $33^{\prime \prime} \times 33^{\prime \prime}$.

Wavelength setting time: as shown in Table 1, the wavelength positioning of the interferometer is very fast ( $\simeq 1.5 \mathrm{~ms}$ ). The instrumental wavelength setting time is therefore imposed by the UBF and it is not constant, depending on the angular rotations of the crystal groups, necessary to set the passband to the demanded wavelength. If then a different wavelength range is requested, also the two filter wheels, preceding the CCD cameras, must be positioned. The maximum setting time amounts therefore to about $1 \mathrm{~s}$; however, in the case of small wavelength steps, as for example in scanning a photospheric line, the setting time typically is $\simeq 0.1 \mathrm{~s}$.

\section{Conclusions}

The Italian Panoramic Monochromator, recently installed at THEMIS, is a post-focus instrument which, preserving all the telescope spatial resolution $\left(\simeq 0.2^{\prime \prime}\right)$, allows to obtain narrow-band (FWHM $=21.5 \mathrm{m \AA}$ at $5500 \AA$ ) monochromatic images of the solar surface with high wavelength stability (better than $10 \mathrm{~ms}^{-1}$ in $10 \mathrm{~h}$ ) and spectral homogeneity of the field $\left( \pm 10 \mathrm{~ms}^{-1}\right)$. Although it is not long that the instrument is operative, some improvements are already under study, to overcome two present instrumental limitations: a too small field of view and a too slow acquisition rate.

In principle, to increase the field, two makeshift solutions are possible. The first one consists in reducing, by means of a suitable optical interface, the effective focal length of the telescope, preserving the relative aperture: in this case the field increases at the expense of the spatial resolution. The second one consists in changing the optics of the instrument to increase the aperture of the beam incident on the interferometer: in this case the field increases at the expense of the spectral resolution. Both these solutions, however, cannot be adopted, due to mechanical constraints.
In the future the present interferometer will be replaced by a new one with a larger diameter, and larger and faster CCDs will be used. This solution will allow to increase the acquisition rate and the field of view, while preserving all the present spatial and spectral resolution.

Acknowledgements. Thanks are due to Prof. A. Righini for his useful suggestions, to Dr. G. Ceppatelli for having written the control software for the instrument, to Mr. G. Falcini and Mr. S. Paloschi for having built the mechanical structure and the electronic controls, to Mr. T. Grisendi and Mr. F. Fabiani for having secured part of the observations performed in Arcetri and for their contribution to the installation at THEMIS. Prof. A. Egidi, Prof. S. Cantarano and Dr. F. Berrilli have built the CCD acquisition systems and written their software; Dr. S. De Muro and the mechanical workshop of the Physics Department of "Tor Vergata" University have designed and built part of the mechanical supports necessary for the installation of the IPM at THEMIS.

This instrument was built with the contribution of the Consiglio Nazionale delle Ricerche (CNR), the Osservatorio Astrofisico di Arcetri, the Università di Firenze and the Università di Roma II.

\section{References}

Bonaccini D., Cavallini F., Ceppatelli G., Righini A., 1989, A\&A 217, 368

Beckers J.M., 1971, Appl. Opt. 10, 973

Beckers J.M., 1972, Appl. Opt. 11, 681

Cantarano S., Cerulli P., Berrilli F., et al., 1993, Mem. Soc. Astron. Ital. 64, 768

Cavallini F., Ceppatelli G., Righini A., et al., 1987, A\&A 184, 386

Cavallini F., Ceppatelli G., Righini A., et al., 1992, Il Nuovo Cimento 15C, 509

Engelhard E.J.G., Bayer-Helms F., 1972, Metrologia 8, 91 